\title{
Software Defined Radio
}

\author{
Archa Sundar ${ }^{1}$, Dhanya $S^{2}$ \\ 1, 2Mahatma Gandhi University, Federal Institute of Science \& Technology, Angamali, Ernakulum, India
}

\begin{abstract}
This paper deals with Software Defined Radio (SDR) is a technology which makes it possible to implement the radio communication process simply with software. Comparing to the traditional radio communication systems, SDR omits all the hardware and replaces them by pure software. This solution also gives a great advantage in flexibility because a SDR receiver is able to decode all the signals. Past decades deals with many communication standards and even today also many researches are going on. A major problem in these are different communication standards use different hardware circuitry which causes these communication standards regional. Inoder to mitigate this problem, many developments are occurred in mobile communication to develop a communication system which works by swapping the software with a single hardware circuitry. The main aim of this paper is to develop a model of a Software defined Radio using MATLAB tool and to simulate the Bluetooth standard. For the Bluetooth standard the basic modulation scheme used is GFSK and Frequency hopping spread spectrum is used.
\end{abstract}

Keywords: SDR, MATLAB, Bluetooth

\section{Introduction}

Radios are essential parts of everyday human communications. The first thing comes into mind when thinks about radio is AM/FM radios in the cars, hand-held two-way radios, or $\mathrm{CB}$ radios. But radios are much more prevalent in society than others. In present life the use radio increases in large quantity, even the Wi-Fi adapters within a computer are radios and Bluetooth earpieces used to talk on the phone are radios. So in general, a radio is a device that can transmits or receives information wirelessly by the use of electromagnetic waves known as radio waves.

Basically, radios have been made from hardware pieces designed to use in one specific radio. These radios can be generally defined as hardware-defined radios since the radio is completely hardware dependent such as electronic devices and electrical circuits .From the emergence of softwaredefined radios, a new route for mobile communication is emerged. SDR are a new type of radio in which the type of radio is determined by a piece of software rather than hardware. These software-defined radios (SDRs) or software radios are a developing technology which assures a dramatic increase in the development of mobile communications with many advantages that make them attractive to researchers and radio developers alike.

\section{Need for Software-Defined Radios}

In the past decades, the area of wireless communications has been developing and emerging in a rapid form. All new electronic devices implement some sort of wireless communications, may be in the form of Wi-Fi, Bluetooth, or cellular technologies like CDMA or LTE. Specific protocols are defined for each of these different radio systems. So different radio systems had to be implemented using hardware configurations.

In hardware radios, physical components are used which cannot be easily modified. This leads to several limitations in this hardware radios. Main problem arising due to this are; first is need of different hardware setups for each radio technology leads to the usage of significant amounts of space, especially if a particular system needs several different radio technologies. Second one is implementation of separate hardware protocols for systems needing many different radio standards becomes expensive. A key example of this is cellular phone technology. There arise several attempts to standardize radio protocol in cellular phone technology by entire nations and regions, but the cell phones still need to support old standards still in use and alternate standards in different regions so that a single phone can operate in many locations. Separate physical systems for each communication standard in current hardware system cause limitations to cell phones which increases both the size and cost of cell phones. Third is hardware radios are not easily updated when new technology is developed. To become faster and more advanced, radio technology and protocols are constantly evolving. This is the reason why a protocol used today could be obsolete in just a few years. Because of all these limitations inherent in static hardware radio systems, there is a need for a different kind of radio system and has been developed within the past few years.

Inorder to solve these hardware problem, engineers decided to implement radio parts using software rather than hardware. Implementation of radio parts using software rather than hardware enables a more easily configured, modified, and developed radio for multiple systems. Radio implementation in this new form came to be known as software defined radio (SDR) or software radio. The development of new form make its goal as implementation of a fully functional radios in one system that previously needed multiple systems. We can say that the migration of hardware-defined radios to software-defined radios corresponds with the move from analog radio systems to digital radio system. The digital communication systems provided some key benefits over the existing analog communication systems. Other than the advantages, both analog and digital communication systems still exist today. Even though analog systems are still used in almost all applications, digital systems will never be able to completely replace analog systems. Similarly, even though the software radios have so many advantages to overtake hardware radios in many situations throughout the coming years, the simplicity and dependability of hardware radios can never 


\section{International Journal of Science and Research (IJSR) \\ ISSN (Online): 2319-7064}

Index Copernicus Value (2013): 6.14 | Impact Factor (2014): 5.611

be replaced and will continue to exist. Nowadays radio systems today are rapidly becoming software defined rather than hardware defined radios.

So Software Defined Radio can be defined as a radio communications transceiver system in which all the typical components of a communication system are present such as mixers, modulators/demodulators, detectors, amplifiers that are implemented through software rather than hardware. The development of SDR leads to the scope of developing a system which is compatible with more than one mobile communication standard. This can be achieved by using reconfigurable hardware and swapping the software for different technologies.

\section{Proposed Model}

The model proposes an SDR system with bluetooth standard, which provides all bluetooth functionalities. It mainly consists of three parts, a transmitter section, a bluetooth section and a receiver section. The input to the system will be audio signal. The given input audio signal is modulated using a BPSK modulator which is then given to the bluetooth block which consists of mainly a frequency hopping, GFSK modulator and FSK modulator. At the receiver the signal is again demodulated to retrieve the original signal. A software defined radio is a transmitter and receiver system that uses digital signal processing (DSP) for coding, decoding, modulation, and demodulation. This allows much more power and flexibility when choosing and designing modulation and coding techniques.

The general block diagram of SDR consists of input to the system as digital data in a computer file. This data will be modulated by the transmitter and sent to the channel. The channel will introduce interference to the signal in the forms of attenuation, phase delay, and noise. At the receiver side, the signal will be demodulated and reconstructed to produce the original transmitted message which is demonstrated in figure 1.

Bluetooth is relatively easy to implement. Frame format for Bluetooth is simple compared to the WLAN protocol and moreover the only thing which is going to be implemented in the Bluetooth is point to point connection and no other complex tasks. Bluetooth standard does not state any source coding since the Bluetooth frame is relatively smaller.

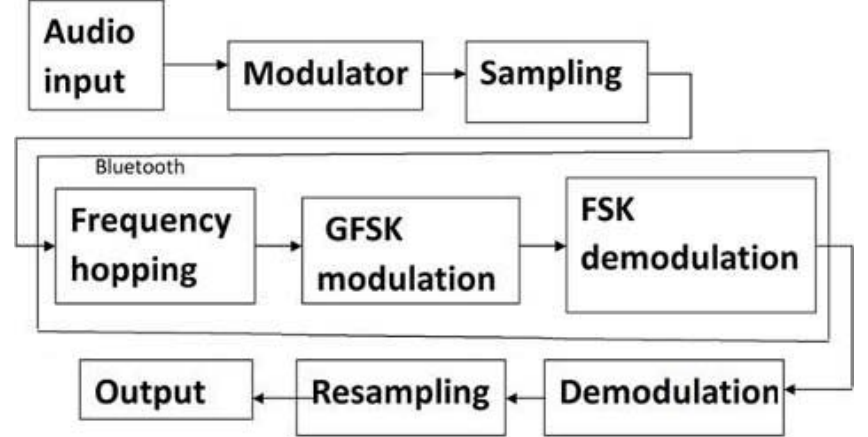

Figure 2: Block diagram of proposed model

The program is completely implemented in MATLAB. The algorithm for the proposed model is shown below:

\subsection{Algorithm}

Step.1: Inputting audio signal

Step.2:Sampling

Step.3: Modulated the sampled signal

Step.4:Generate 6 carrier frequencies for spreading

Step.5:Generate frequency hops by concatenating each frequencies

Step.6:Spread the modulated signal using these frequency hops

Step.7:Spread ed signal is given for GFSK modulation Step.8:Signal is passed through AWGN channel Step.9:Demodulate the signal using FSK demodulation Step.10:Reconstruct the signal by resampling Step.11:Compare both input and output signals by measuring the MSE value

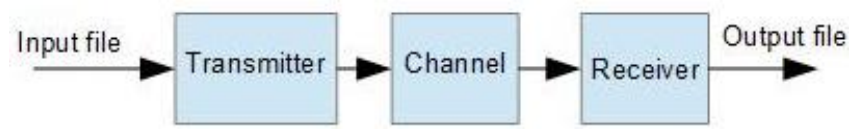

Figure 1: Basic block diagram of SDR

In this paper, we modeled the proposed system as SDR with bluetooth standard. The input to the system will be audio signal. The given input audio signal is modulated using a BPSK modulator which is then given to the bluetooth block which consists of mainly a frequency hopping, GFSK modulator and FSK modulator which is shown in figure 2 ..

\section{Results and Discussions}

The proposed model consists of mainly three parts, a transmitter, bluetooth section and a receiver. The transmitter section consists of input to the system as an analog audio signal, which is of very small duration as shown in figure 3. The processing of the signal is done in binary form. So the analog signal is first converted into binary form. The obtained signal is then BPSK modulated so that a phase shift in the modulated signal occurs as shown in figure 4. 


\section{International Journal of Science and Research (IJSR) \\ ISSN (Online): 2319-7064}

Index Copernicus Value (2013): 6.14 | Impact Factor (2014): 5.611

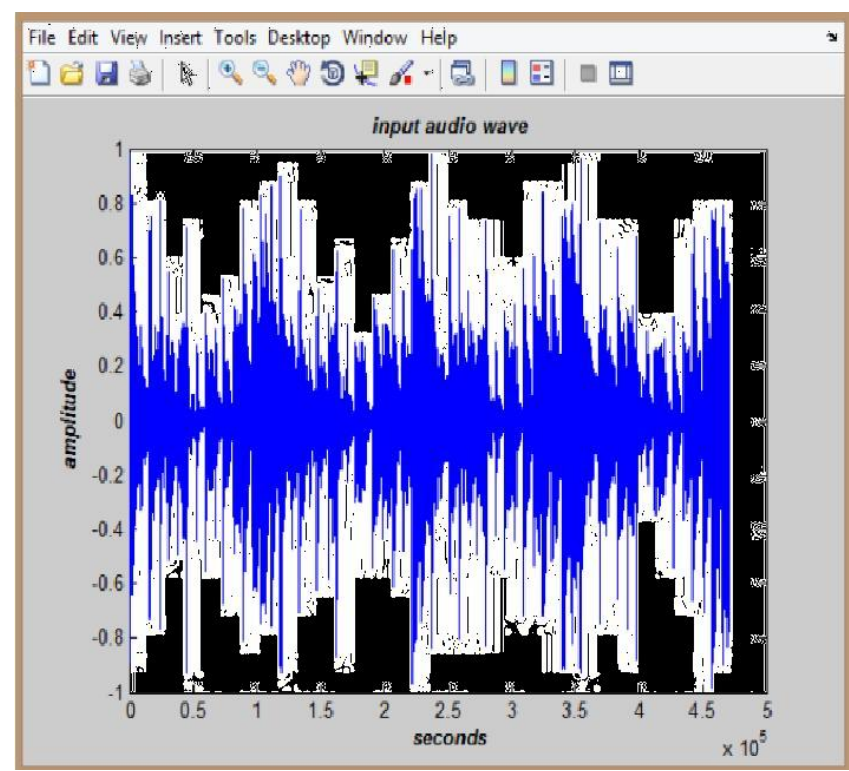

Figure 3: Input audio signal

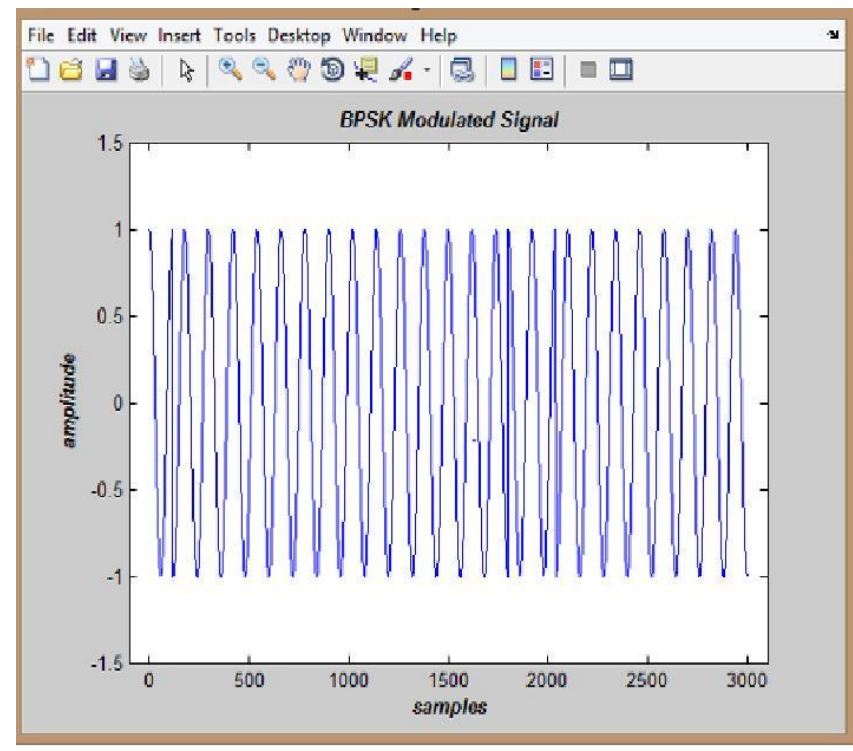

Figure 5: Spread ed BPSK signal

Next is the bluetooth part. As we know it consists of different parts in which all are separately implemented. Initially generate six frequencies randomly, which is used for spreading the signal. After generating these frequencies concatenate each one by one to get the complete signal. Since these frequencies are used for frequency hopping, they are called hopping sequences and thats why the signal thus generated is called frequency hopping spread signal. The spread ed signal is shown in figure 5 .

By using these spread ed frequencies the BPSK modulated signal is spread ed which is shown in figure 6. Using the six hopping sequences frequency hopped spread-spectrum signal is generated. The signal switches carrier channels rapidly, at a rate of 1600 hops per second, over a determined pattern of channels.

The frequency hopped spread spectrum signal is then given for GFSK modulation, which includes a Gaussian filter and FSK modulation. Frequency Hopping Spread Spectrum (FHSS) is a radio signals transmitting method by rapidly switching a carrier among many frequency channels, using a pseudo random sequence known to both transmitter and
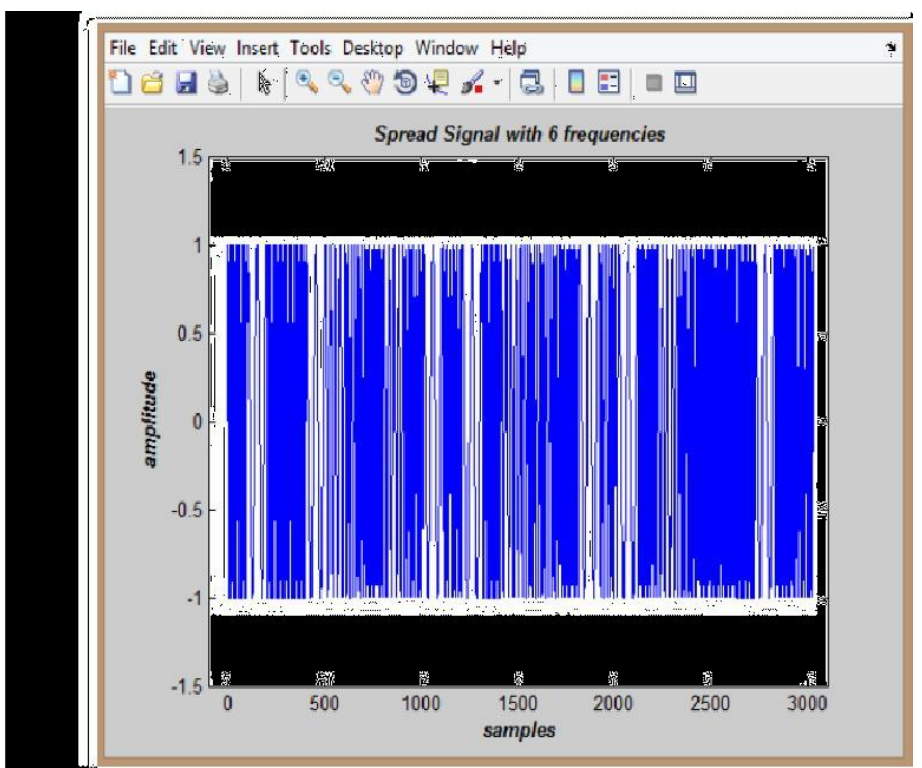

Figure 4: BPSK modulated signal

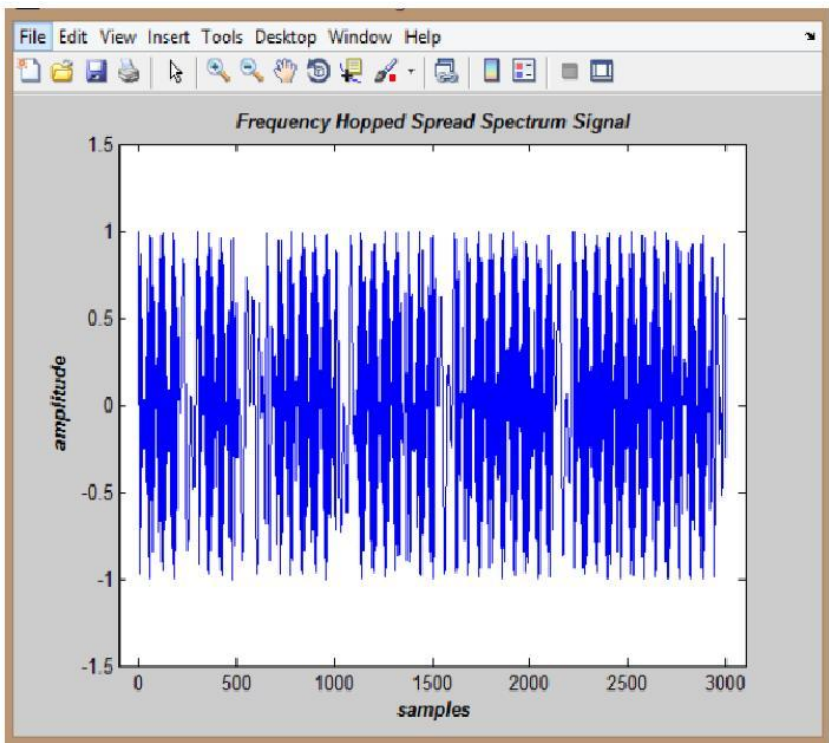

Figure 6: Frequency hopped spread-spectrum

receiver. Signal is passed through a Gaussian filter inorder to remove Gaussian noise from it, and is FSK modulated. The figure 7 shows the FSK modulated filtered signal.

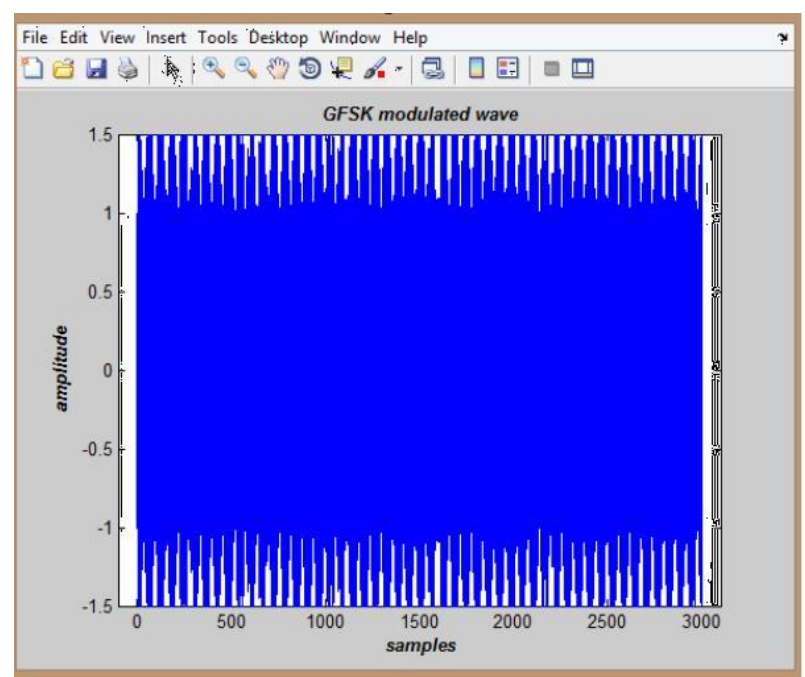

Figure 7: GFSK modulated signal

Volume 5 Issue 1, January 2016 


\section{International Journal of Science and Research (IJSR) \\ ISSN (Online): 2319-7064 \\ Index Copernicus Value (2013): 6.14 | Impact Factor (2014): 5.611}

The GFSK modulated signal is then passed through a channel which is corrupted with noise. Last part of the bluetooth section is FSK demodulation, which is shown in the figure 8 .

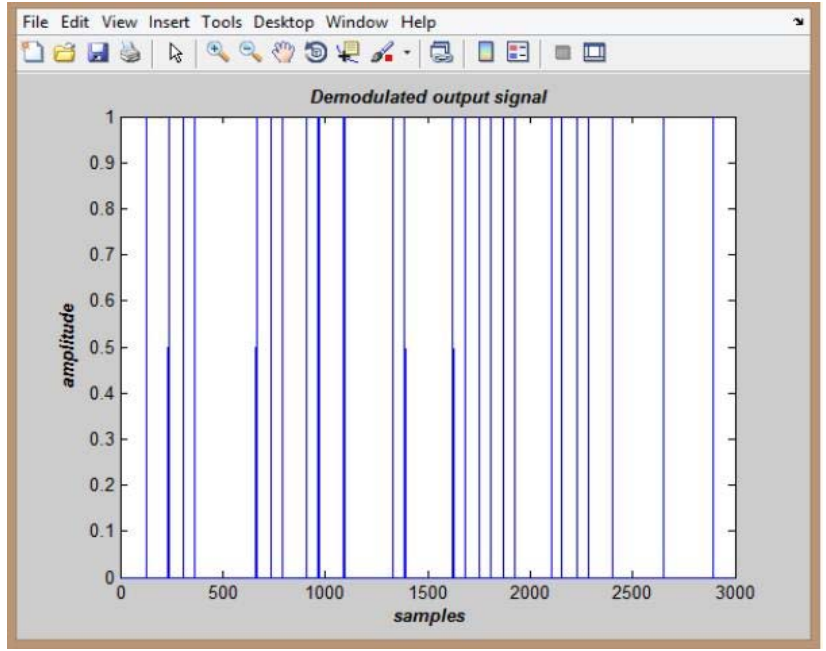

Figure 8: GFSK demodulated signal

At the receiver side, the signal is demodulated again and converted back to audio signal.

\section{Comparison between input and Output audio}

Even though an audio signal is transmitted through an SDR with bluetooth, inorder to check whether the input and output audio are same, we calculate the mean square error (MSE), which can be expressed as $\mathrm{MSE}=(\operatorname{sum}(\operatorname{abs}($ (inputoutput).^2)) $) /($ length(input))

i.e, here $\mathrm{MSE}=(\operatorname{sum}(\operatorname{abs}(($ wave-

output $\left.\left.\left.){ }^{\wedge} 2\right)\right)\right) /($ length $($ wave $))=0.0001$

\section{Advantages}

The advantages of SDR include reduced component cost because hardware specific components are replaced by DSPs and FPGAs. The number of components needed tends to be lower. DSP components can compensate for problems in other areas of the system. The main difference between traditional radios and software defined radios is that these SDRs senses their environment and adapt to it. This is important particularly to government agencies such as the FCC who is in the process of reallocating spectrum usage in the TV bands. There's a shift away from rigid, spectrum allocations to a demand-based approach to maximum the usage in that band.

\section{Conclusion and Future Scope}

A pure software platform based transceivers for the communication standard can be implemented in this architectures. This implementation makes the differences between the equipment currently in the present day market very narrow. The equipment built using the SDR platform will be greatly benefited for users as they support multiple communication standards with the same given hardware.
There will be no need of buying multiple equipments for multiple purposes as one equipment can perform and take care of all the things. Active research is being carried out in this area and much commercial SDR equipment for different applications is available for commercial use. A disadvantage of this is their costs are presently very huge. In the future, we can expect all the mobiles, talkies that are used will become more and more flexible and tend towards SDR platform rather than the traditional architectures being used now. We can also use the advantages and advancements made in VLSI and the microprocessors fields to make a better architecture of an SDR. A day will come soon where a single transceiver can act as all in one and can be used as a mobile phone which can support both GSM and CDMA, which can also be connected to a WLAN access point, which has got Bluetooth connectivity, which can receive FM signals and work as FM radio etc.

Active research are being carried out in this area and much commercial SDR equipment for different applications to make available for commercial use. With the advancements made in VLSI and the microprocessors fields the architecture of an SDR becomes simplified rapidly May be a single transceiver can act as all in one in future.

\section{References}

[1] "ereftware Defined Radio Implementation (With simulation \& analysis) $)^{\text {eee }}$ Shriram K Vasudevan, International Journal of Computer Applications published in 2010

[2] "A General System Design \& Implementation of Software Defined Radio System eece, Journal of Information, Knowledge and Research in Electronics and Communication Engineering, volume 02, Nov 12Oct 13,J.H. Varde, N.B. Gohil, J.H.Shah

[3] "Software Defined Radio"ee - Basics and Evolution to Cognitive Radio,EURASIP Journal on Wireless Communication and Networking 2005 Friedrich K. Jondral

[4] "Published paper in Computer "Communications" titled "Software Radio Reconfiguration Management". By Klaus Moessner, Didier Bourse, Dieter Greifendorf and Joerg Stammen dated 26th February 2002.

[5] "Bluetooth protocol Architecture" by Riku Mettala.

\section{Author Profile}

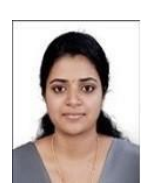

Archa Sundar is pursuing M-Tech on Communication Engineering at FISAT, Angamali, Mookkanoor, Ernakulam, Kerala, India. Completed BTech on Electronics and Communication in the year 2010-2014 from College of Engineering, Attingal, Thiruvananthapuram, Kerala, India. She completed plus two from Model Higher Secondary School, Attingal and tenth grade from Sree Vidhyadhiraja English Medium Higher Secondary School in the years 2010 and 2008 respectively.

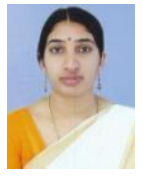

Dhanya. $\mathrm{S}$ is working as Assistant Professor at FISAT, Angamali, Mookanoor, Ernakulam, Kerala, India. She completed M-Tech on Communication Engineering at FISAT with 1st Rank in MG University, Angamali, Mookkanoor, Ernakulam, Kerala, India 